\title{
Validation of Bipartite Network Model of Dengue Hotspot Detection in Sarawak
}

\author{
Woon Chee Kok and Jane Labadin \\ Faculty of Computer Science and Information Technology, Universiti Malaysia Sarawak, \\ Malaysia \\ woonchee.kok@gmail.com, ljane@unimas.com
}

\begin{abstract}
This paper presents the verification and validation processes in producing a realistic bipartite network model to detect dengue hotspot in Sarawak. Based on the result of previous published work, ranking of location nodes of possible dengue hotspot at Sarawak are used to illustrate the validation by comparing the Spearman rank correlation coefficients (SRCC) between the network models. UCINET 6 is used to generate a benchmark ranking result for model verification. A centrality measure analysis feature available in UCINET is used to determine the node centrality of a network model. The validation results show strong ranking similarity for all three groups of network models with good Spearman rank correlation coefficients values of $1.000,0.8000$ and $0.8824(\rho>0.80 ; p<0.001)$ respectively. The top-ranked locations are seen as dengue hotspots and this study demonstrate a new approach to model dengue transmission at district-level by locating the hotspots and prioritizing the locations according to vector density.
\end{abstract}

Keywords: Bipartite Network, Dengue Hotspot, Validation, Verification

\section{Introduction}

Hotspot detection is also referred to as geospatial patterns modeling, area clustering modeling or spatial structure modeling. Typical studies of hotspots detection aim to map the disease occurrences with demographic and environmental properties to better understand the spread of disease. Hotspot detection studies are commonly carried out on infectious diseases such as tuberculosis, dengue and others [1]. Researchers are interested to identify specific environmental properties that are highly correlated to the distribution of the dengue patients in the area with unusually elevated response and thus can be categorized as hotspot.

In a disease domain, it is important for public health authorities to assess whether there is an unusual spatial aggregation of disease cases for them to make decision in allocating resources for prevention. Environmental properties relate to hotspot detection studies in which the physical characteristics of a site and the distribution of the dengue occurrences are included [2]. 
Significant environmental properties are identified and were used to quantify the nodes in the network model from our previous study [3]. Altitude, temperature, precipitation and humidity and temperature are identified as important parameters to quantify the link between the nodes. Dengue vectorial capacity $(V C)$ was defined by [6] shown in equation 1 to describe dengue vector's ability to spread the disease among humans and takes into account host, virus and vector interactions where $m$ represents vector density, $b$ represents transmission probability, $c$ represents infection probability, $a$ represents number of bites, $p$ represents vector survival rate and $n$ represents the extrinsic incubation period (EIP). The parameters involved in the equation are significant predictors to describe the abundance and characteristics of the dengue vector at one hotspot from biological perspectives.

$$
V C=\frac{m b c a^{2} p^{n}}{-\ln p}
$$

The EIP is the viral incubation period between the time when a mosquito takes a blood meal and the time when that mosquito become infectious [4]. In other words, little time is required for the virus to replicate after the mosquito took a blood meal from an infected person and spread through the body of the mosquito until the virus can be passed on to another host. Knowledge about the EIP of dengue for Ae. Aegypti is scarce. Thus, life cycle duration is introduced to examine the risk of dengue occurring in a given area $[2,4-5]$.

The transmission of dengue is strongly dependent on mosquito biting behavior and this biting behavior is temperature-dependent [6-8]. The biting rate can be defined as the number of blood-feeding of a mosquito taken per unit time [9]. Vector biting which is associated with temperature was applied in this study.

Vector survival has long been recognized as one of the most significant parameters in mosquito-borne disease transmission [2,4-5]. Experiments conducted on female Aedes mosquitoes over the temperature range between $15^{\circ} \mathrm{C}$ to $34^{\circ} \mathrm{C}$ revealed that the survival rate are ranged from $23.5 \%$ to $67 \%$ per day and this parameter is used in this study [5].

As a summary, life cycle, survival, biting behavior and frequency of mosquito visits the location are possible important model predictors that contribute to dengue spread.

This paper presents our continuous research in applying bipartite network modeling approach to predict dengue hotspot in Sarawak [3]. Model verification and validation process of the bipartite dengue contact (BDC) network is highlighted in this paper.

\section{$2 \quad$ Materials and methods}

Model verification is a procedure of determining if the implementation of a model has been done correctly [10]. It is also reported as the process to answer the question of "have we built the model right?" [11]. The verification is conducted through benchmark verification. Benchmark verification aims to verify research procedures by 